\title{
Quasilinear parabolic equations and the Ricci flow on manifolds with boundary
}

\author{
Artem Pulemotov \\ Department of Mathematics \\ The University of Chicago \\ 5734 S. University Ave., Chicago, IL 60637-1514, USA \\ Email: artem@math.uchicago.edu
}

\begin{abstract}
The first part of the paper discusses a second-order quasilinear parabolic equation in a vector bundle over a compact manifold $M$ with boundary $\partial M$. We establish a short-time existence theorem for this equation. The second part of the paper is devoted to the investigation of the Ricci flow on $M$. We propose a new boundary condition for the flow and prove two short-time existence results.
\end{abstract}

\section{Introduction}

The present paper is motivated by the desire to investigate geometric evolutions on a compact manifold $M$ with boundary $\partial M$. Our first goal is to study a second-order quasilinear parabolic equation in a vector bundle over $M$. We then apply the obtained results to the analysis of the Ricci flow on $M$. Let us explain the essence and the history of the problems to be considered.

A significant step in the investigation of geometric evolutions on $M$ is to acquire information about second-order quasilinear parabolic equations in vector bundles over $M$. Particularly, it is important to have a short-time existence theorem that would cover a wide range of boundary conditions and produce a solution with ample differentiability properties. Until now, such a theorem did not appear in the literature. Even in the case where $M$ is the closure of a domain in $\mathbb{R}^{n}$, there was no published result that would meet the demands of the applications to geometric evolutions. In Section 2 of the present paper, we make an effort to remedy this situation. We establish a short-time existence theorem for a second-order quasilinear parabolic equation in a vector bundle over $M$. No assumptions are imposed on the geometry of $M$. Yet even in the the case where $M$ is the closure of a domain in $\mathbb{R}^{n}$, our result is somewhat different from the results that previously appeared in print. We will now describe it in more detail.

Fix a Riemannian metric on $M$. Let $E$ be a vector bundle over $M$. Suppose $E$ is equipped with a fiber metric and a connection $\nabla$. We focus on the equation

$$
\frac{\partial}{\partial t} u(x, t)-H^{i j}(u(x, t), t) \nabla_{i} \nabla_{j} u(x, t)=F(u(x, t), \nabla u(x, t), t)
$$

for a section $u$ of $E$ depending on $t \geq 0$. Here, $H$ is a smooth map from $E \times[0, \infty)$ to the space of symmetric $(2,0)$-tensors over $M$, and $F$ is a smooth map from $E \times\left(T^{*} M \otimes E\right) \times[0, \infty)$ to $E$. The meaning of the rest of the notation should be easy to infer from the context. In the beginning of Section 2, we explain it pedantically. Suppose now that $E_{\partial M}$ is the restriction of the bundle $E$ to $\partial M$ and $W$ is a subbundle of $E_{\partial M}$. We supplement equation (1.1) with the boundary conditions

$$
\begin{aligned}
\operatorname{Pr}_{W} u(x, t) & =o(x), \\
\operatorname{Pr}_{W^{\perp}}\left(H^{i j}(u(x, t), t) \nu_{i}(x) \nabla_{j} u(x, t)\right) & =\Psi(u(x, t), t) .
\end{aligned}
$$


Here, $o$ is the zero section of $E$, and $\nu$ is the outward unit normal covector field on $\partial M$. The smooth map $\Psi$ acts from $E_{\partial M} \times[0, \infty)$ to $W^{\perp}$. The first line in (1.2) should be thought of as the Dirichlet boundary condition. It is imposed on $u$ inside $W$. The second line in (1.2) may be looked at as a nonlinear nonhomogeneous Neumann condition. It is imposed inside $W^{\perp}$. Finally, we supplement (1.1) with the initial condition

$$
u(x, 0)=u_{0}(x) .
$$

In this formula, $u_{0}$ is a smooth section of $E$. The main result of Section 2 requires two additional assumptions. First, we demand that equation (1.1) be parabolic. Second, we impose a compatibility condition near $\partial M$ when $t=0$. If these assumptions are satisfied, the main result of Section 2 tells us that problem (1.1)-(1.2)(1.3) has a solution on $M \times[0, T)$ for some $T>0$. This result appears below as Theorem 2.1. We point out that the solution it produces is smooth on $M \times(0, T)$. A problem akin to (1.1)-(1.2)-(1.3) was studied in W.-X. Shi's paper 24]. That work, however, only allowed Dirichlet-type boundary conditions. It turns out that the nonlinearities in the second line of (1.2) contribute substantially to the difficulty of the question of the existence of solutions to (1.1)-(1.2)-(1.3).

Let us assume for a moment that the manifold $M$ is the closure of a domain in $\mathbb{R}^{n}$ and $E$ is the product $M \times \mathbb{R}^{d}$ carrying the standard fiber metric and connection. Note that, even under these assumptions, it is possible for $W$ to be a nontrivial bundle. We encounter such a phenomenon when dealing with the Ricci flow later in the present paper. Suppose for now, however, that $W=\partial M \times \mathcal{R}$ with $\mathcal{R}$ being the space of all $\left(e_{1}, \ldots, e_{d}\right) \in \mathbb{R}^{d}$ such that $e_{d^{\prime}+1}=\ldots=e_{d}=0$ for a fixed $d^{\prime}$ between 0 and $d$. In this case, equation (1.1) may be viewed as a second-order quasilinear system. The section $u$ takes the form $u=\left(u^{1}, \ldots, u^{d}\right)$ with each $u^{m}$ real-valued. Formulas (1.2) become Dirichlet boundary conditions on $u^{1}, \ldots, u^{d^{\prime}}$ and nonlinear nonhomogeneous Neumann conditions on $u^{d^{\prime}+1}, \ldots, u^{d}$. In the case where $M$ is the closure of a domain in $\mathbb{R}^{n}, E=M \times \mathbb{R}^{d}$, and $W=\partial M \times \mathcal{R}$, problems similar to (1.1)-1.2 (1.3) were extensively studied. Several different methods were proposed to prove the existence of solutions. For example, the papers [2, 3 ] by H. Amann used abstract functional-analytic techniques. The work [30] by P. Weidemaier employed a more straightforward fixed-point argument in a Sobolev-type space. The reader should see [15, 12, 1] for other approaches.

No major restrictions are imposed in Section 2 on the geometry of $M, E$, or $W$. But even in the case where $M$ is the closure of a domain in $\mathbb{R}^{n}, E=M \times \mathbb{R}^{d}$, and $W=\partial M \times \mathcal{R}$, the material we present there did not previously appear in the literature in the same form. When we restrict our attention to this geometrically trivial situation, the theorem in the introduction of H. Amann's paper $[3$ is somewhat similar to our Theorem 2.1. However, that result involves a different compatibility condition. We should remark that the arguments in [3] are rather complicated. It seems that adapting them to the setting of manifolds and vector bundles would be a tedious task. When $M$ is the closure of a domain in $\mathbb{R}^{n}, E=M \times \mathbb{R}^{d}$, and $W=\partial M \times \mathcal{R}$, the reasoning in P. Weidemaier's paper [30 is akin to much of our reasoning in Section 2. On the other hand, the work [30] is concerned with a narrower range of boundary conditions. Besides, it does not touch upon the issue of the smoothness of solutions.

As we previously declared, our investigation of second-order quasilinear parabolic equations was motivated by the desire to study geometric evolutions on manifolds with boundary. Let us say a few words about the specific applications of Theorem 2.1. In Section 3, we establish two short-time existence results for the Ricci flow on a manifold with boundary. Theorem 2.1 is a crucial ingredient in our considerations. The paper [6] studies the Yang-Mills heat flow on 2- and 3-dimensional manifolds with boundary and utilizes it for the purposes of quantum field theory. Among other things, the authors of [6] prove the existence of solutions to the flow; see also 20. We speculate that Theorem 2.1 can be used to simplify their arguments. More applications of this nature may emerge in the near future. Theorem 2.1 seems to be a convenient tool for proving the existence of solutions to various geometric evolutions.

Section 3 of the present paper focuses on the Ricci flow on the manifold $M$. Our goal is to introduce a new boundary condition for the flow and establish two short-time existence results. More precisely, consider the equation

$$
\frac{\partial}{\partial t} g(x, t)=-2 \operatorname{Ric}^{g}(x, t)
$$

for a Riemannian metric $g$ on $M$ depending on the parameter $t \geq 0$. We supplement (1.4) with the initial 
condition

$$
g(x, 0)=\hat{g}(x)
$$

Here, $\hat{g}$ is a smooth Riemannian metric on $M$. Equation (1.4) is the Ricci flow equation on $M$. To learn about its history, intuitive meaning, technical peculiarities, and geometric applications, the reader should refer to the many quality books on the subject, such as $[8,9,29$, 17. Examples of how it comes up in mathematical physics may be found in [13, 18, 14, 19] and other papers. One more interesting application is to the regularization of non-smooth Riemannian metrics; see, e.g., [25, 26].

The Ricci flow on manifolds with boundary is not yet deeply understood. The root of all evil lies in the fact that equation (1.4) is not parabolic. For this reason, it is difficult to find geometrically meaningful and analytically appealing boundary conditions for solutions of (1.4) -(1.5). It would be natural to demand, for example, that the metric induced by $g$ on $\partial M$ always coincide with the metric induced by $\hat{g}$. But so far, nobody knows how to prove the short-time existence of solutions under such a requirement. Progress towards finding boundary conditions to go with (1.4) - (1.5) was made by Y. Shen in his dissertation 22. Those results were also published in the paper 23 . Y. Shen considered the case where the second fundamental form II of the boundary with respect to $\hat{g}$ satisfied the equality $\hat{\mathrm{II}}(x)=\tau \hat{g}(x)$ with $\tau \in \mathbb{R}$ for all $x \in \partial M$. In other words, he assumed that $\partial M$ was umbilid 1 when $t=0$. He was then able to prove the existence of $T>0$ and a solution $g$ to problem (1.4) (1.5) on $M \times[0, T)$ such that the second fundamental form II of $\partial M$ with respect to $g$ satisfied the equality

$$
\mathrm{II}(x, t)=\tau g(x, t)
$$

for all $x \in \partial M$ and $t \in[0, T)$. Not much is known about the behavior of $g$ for large $t$. This question was addressed in [11] under additional assumptions. We should point out that the case where $\tau=0$ is somewhat special. If $\tau=0$, then $\partial M$ is totally geodesic with respect to $\hat{g}$. In this situation, one can say a few things about how the solution $g$ produced in [22] behaves for large $t$; see [22, 23, 7].

No new boundary conditions for the Ricci flow in dimensions higher than 2 have been proposed in the literature since the publication of Y. Shen's dissertation. A certain amount of work, however, has been done on surfaces. The list of relevant texts includes 16, 15, 10. While not much is known today about the Ricci flow on manifolds with boundary, it is clear that results in this area would have significant geometric applications. They would also be useful to mathematical physicists; see [13, 14.

In section 3, we propose a new boundary condition on the solutions of the Ricci flow and prove two short-time existence results, a theorem and a proposition. Let $\hat{\mathcal{H}}$ be the mean curvature of $\partial M$ with respect to $\hat{g}$. Our theorem assumes that $\hat{\mathcal{H}}$ is equal to a constant $\mathcal{H}_{0} \in \mathbb{R}$ everywhere on $\partial M$. It then claims that there exist $T>0$ and a solution $g$ to problem (1.4) (1.5) on $M \times[0, T)$ such that the boundary condition

$$
\mathcal{H}(x, t)=\mu(t) \mathcal{H}_{0}
$$

holds for all $x \in \partial M$ and $t \in[0, T)$. Here, $\mu$ is a function that may be thought of as a normalization factor, and $\mathcal{H}$ is the mean curvature of $\partial M$ with respect to $g$. Our proposition touches upon the question of the behavior of the Ricci flow on manifolds with convex boundary. This question is natural, and it is related to some of the material in [22, 23, 11, 4]. Among other things, our proposition implies that, if $\partial M$ is convex with respect to $\hat{g}$, we can find $T>0$ and a solution $g$ of (1.4)-(1.5) on $M \times[0, T)$ such that $\partial M$ remains convex with respect to $g$.

The results in Section 3 constitute a step towards understanding the Ricci flow on manifolds with boundary. We suspect they can also be used for the purposes of regularizing non-smooth Riemannian metrics on such manifolds. It is worth mentioning that the proofs of the results in Section 3 are based on the method commonly known as DeTurck's trick. These proofs rely substantially on Theorem 2.1 .

\section{Parabolic equations in vector bundles}

Suppose $M$ is a smooth $n$-dimensional $(n \geq 2)$ manifold with boundary. We assume that $M$ is compact, connected, and oriented. The notations $M^{\circ}$ and $\partial M$ will be used for the interior and the boundary of $M$.

\footnotetext{
${ }^{1}$ There is ambiguity in the literature as to the use of the term "umbilic" in this context. See the discussion in [1].
} 
Consider a smooth vector bundle $E$ over $M$ with projection $\pi$ and standard fiber $\mathbb{R}^{d}$. We will discuss second-order quasilinear parabolic equations for sections of $E$ subject to nonlinear nonhomogeneous boundary conditions. Our goal will be to establish the short-time existence of solutions. This result will later help us investigate the Ricci flow.

Throughout Section 2, we fix a smooth Riemannian metric on $M$. The tangent bundle $T M$ is equipped with the Levi-Civita connection. The letter $\nu$ will stand for the outward unit normal covector field on $\partial M$. Let us also fix a smooth fiber metric in $E$ and a smooth connection in $E$ compatible with this metric.

Our arguments will involve tensor products of the form $\mathfrak{E}=\mathfrak{E}_{1} \otimes \cdots \otimes \mathfrak{E}_{k}$ with $\mathfrak{E}_{i}$ equal to $T M, T^{*} M$, or $E$ for each $i=1, \ldots, k$. Here, $T^{*} M$ designates the cotangent bundle, and $k$ is a natural number. The Riemannian metric on $M$ and the fiber metric in $E$ generate a fiber metric in every such $\mathfrak{E}$. Given $\eta \in \mathfrak{E}$, we write $|\eta|$ for its norm. The Levi-Civita connection in $T M$ and the fixed connection in $E$ give rise to a connection in $\mathfrak{E}$. We write $\nabla f$ for the covariant derivative of a section $f$ of $\mathfrak{E}$. Our considerations will also involve second-order differential operators. In particular, $\nabla \nabla f$ stands for the second covariant derivative of $f$.

We will sometimes employ local coordinates on the manifold $M$. Let us introduce the corresponding notation. In what follows, we implicitly assume that a coordinate system $\left\{x_{1}, \ldots, x_{n}\right\}$ is chosen in a neighborhood of every point $x \in M$. If $T$ is a $(k, l)$-tensor at $x$, we write $T_{j_{1} \ldots j_{l}}^{i_{1} \ldots i_{k}}$ for its components in this coordinate system. Given a section $f$ of the bundle $\mathfrak{E}$, the notation $\nabla_{i} f$ stands for its covariant derivative in the direction of $\frac{\partial}{\partial x_{i}}$. Analogous shorthand is used for second-order operators. Namely, $\nabla_{i} \nabla_{j} f$ means $\nabla \nabla f$ applied to $\frac{\partial}{\partial x_{i}}$ and $\frac{\partial}{\partial x_{j}}$. The Einstein summation convention is in effect.

\subsection{Formulation of the existence theorem}

Our purpose is to study the solvability of second-order quasilinear parabolic equations for sections of $E$ subject to nonlinear nonhomogeneous boundary conditions. To begin with, consider a smooth mapping

$$
H: E \times[0, \infty) \rightarrow T M \otimes T M .
$$

We assume that $H(\eta, t)$ is a symmetric tensor over $\pi(\eta)$ for all $\eta$ and $t$. It will be necessary to impose one more requirement on $H$, but we postpone this until a little later. Meanwhile, consider another smooth mapping

$$
F: E \times\left(T^{*} M \otimes E\right) \times[0, \infty) \rightarrow E .
$$

We demand that $\pi(F(\eta, \theta, t))=\pi(\eta)$ for all values of $\eta, \theta$, and $t$. Our attention will be focused on the equation

$$
\frac{\partial}{\partial t} u(x, t)-H^{i j}(u(x, t), t) \nabla_{i} \nabla_{j} u(x, t)=F(u(x, t), \nabla u(x, t), t), \quad x \in M^{\circ}, t \in(0, T),
$$

for a section $u$ of $E$ depending on the parameter $t \in[0, T)$ with $T>0$. The first step is to supplement (2.1) with boundary conditions.

Let $E_{\partial M}$ denote the set of all $\eta \in E$ such that $\pi(\eta) \in \partial M$. This set has the structure of a vector bundle over $\partial M$ induced by the structure of $E$. Also, $E_{\partial M}$ inherits the fiber metric from $E$. Suppose $W$ is a subbundle of $E_{\partial M}$. Let $W^{\perp}$ be the orthogonal complement of $W$ in $E_{\partial M}$. Introduce a smooth mapping

$$
\Psi: E_{\partial M} \times[0, \infty) \rightarrow W^{\perp} .
$$

It is assumed that $\pi(\Psi(\eta, t))=\pi(\eta)$ for all values of $\eta$ and $t$. We impose the boundary conditions

$$
\begin{aligned}
\operatorname{Pr}_{W} u(x, t) & =o(x), \\
\operatorname{Pr}_{W^{\perp}}\left(H^{i j}(u(x, t), t) \nu_{i}(x) \nabla_{j} u(x, t)\right) & =\Psi(u(x, t), t), \quad x \in \partial M, t \in(0, T),
\end{aligned}
$$

on the solutions of (2.1). Here, $\operatorname{Pr}_{W}$ and $\operatorname{Pr}_{W^{\perp}}$ stand for the orthogonal projections in $E_{\partial M}$ onto $W$ and $W^{\perp}$. The letter $o$ refers to the zero section of $E$. In essence, we impose the Dirichlet boundary condition on $u$ inside $W$ and a nonlinear nonhomogeneous Neumann condition inside $W^{\perp}$. To understand 
what formulas (2.2) mean in a geometrically trivial case, the reader may revisit the introduction to the present paper.

Suppose $u_{0}$ is a smooth section of $E$. We supplement equation (2.1) with the initial condition

$$
u(x, 0)=u_{0}(x), \quad x \in M .
$$

Our goal is to establish the solvability of problem (2.1)-(2.2) - (2.3). In order to do so, we need two additional assumptions. The first one is a parabolicity condition on equation (2.1). We suppose there is a constant $c_{1}>0$ such that the inequality

$$
H^{i j}(\eta, t) \xi_{i} \xi_{j} \geq c_{1}|\xi|^{2}
$$

holds for every $\eta \in E, t \in[0, \infty)$, and $\xi \in T^{*} M$ projecting on $\pi(\eta)$. The reader should see, e.g., [29, Chapter 4] for an elaborate discussion of the concept of parabolicity in the framework of vector bundles. The second assumption is the natural compatibility condition

$$
\begin{aligned}
\operatorname{Pr}_{W} u_{0}(x) & =o(x), \\
\operatorname{Pr}_{W^{\perp}}\left(H^{i j}\left(u_{0}(x), 0\right) \nu_{i}(x) \nabla_{j} u_{0}(x)\right) & =\Psi\left(u_{0}(x), 0\right), \quad x \in \partial M .
\end{aligned}
$$

It is now time to state the main result of Section 2, This result is an existence theorem for problem (2.1)(2.2) -(2.3). It will be utilized in Section 3 when we investigate the Ricci flow.

Theorem 2.1. Consider the initial-boundary value problem (2.1)-(2.2)-(2.3). Suppose the parabolicity condition (2.4) and the compatibility condition (2.5) are satisfied. Then there exist a number $T>0$ and a map $u: M \times[0, T) \rightarrow E$ such that the following requirements are met:

1. The equality $\pi(u(x, t))=x$ holds for every $x \in M$ and $t \in[0, T)$. In other words, $u$ is a section of $E$ depending on $t \in[0, T)$.

2. The map $u$ and the covariant derivative $\nabla u$ are continuous on $M \times[0, T)$. Furthermore, $u$ is smooth on $M \times(0, T)$.

3. Equalities (2.1), (2.2), and (2.3) hold for $u$.

Remark 2.2. The number $T>0$ whose existence the theorem asserts is dependent on the mappings $H, F$, $\Psi$, and $u_{0}$. It may also be affected, for example, by the Riemannian metric on $M$.

Remark 2.3. Suppose $\Xi$ is an open neighborhood of the set $\left\{u_{0}(x) \mid x \in M\right\}$ in $E$. It is not difficult to verify that the theorem still holds if the mappings $H, F$, and $\Psi$ are only defined on $\Xi \times[0, \infty), \Xi \times\left(T^{*} M \otimes E\right) \times[0, \infty)$, and $\left(\Xi \cap E_{\partial M}\right) \times[0, \infty)$. Furthermore, let $\Omega$ be the set of $(\eta, \theta, t) \in \Xi \times\left(T^{*} M \otimes E\right) \times[0, \infty)$ such that $\eta$ and $\theta$ project onto the same point in $M$. The theorem prevails if $F$ is only defined on $\Omega$.

Remark 2.4. It may be possible to improve the regularity of $u$ on $M \times[0, T)$ by imposing higher-order compatibility conditions along with (2.5); cf. [15, pages 319-321]. But this issue remains beyond the scope of the present paper.

Remark 2.5. One may be able to prove an analogue of Theorem 2.1 in a more general setting. Namely, suppose End $E$ is the bundle of endomorphisms of $E$ and the mapping $H$ acts from $E \times[0, \infty)$ to $T M \otimes$ $T M \otimes$ End $E$ instead of $T M \otimes T M$. It is clear how problem (2.1)-(2.2)-(2.3) should be modified in this case. It may then be possible to establish an existence result analogous to Theorem 2.1. But we do not concern ourselves with this in the present paper. Let us just mention that the references [27, 2, 12, 28, 3, 30, might be helpful.

Before we can prove Theorem 2.1, we need to introduce additional notation, make a few comments, and state a lemma. This will be done in Sections 2.2 and 2.3. When the preparations are finished, we will use a fixed-point argument to produce $T$ and $u$. The last step will be to establish the smoothness of $u$ by localizing our equation and appealing to some classical facts from [15]. 


\subsection{Spaces of vector bundle sections}

We will deal with a multitude of spaces of vector bundle sections. Although some of these spaces are rather classical, they can be approached from several different viewpoints. In order to exclude ambiguity, and for the convenience of the reader, we will outline the definitions with which we will work in this paper.

Let us use the notation $\mathbb{R}_{+, 0}^{n}$ for the open half-space $\left\{\left(y_{1}, \ldots, y_{n}\right) \in \mathbb{R}^{n} \mid y_{n}>0\right\}$ and the notation $\mathbb{R}_{+}^{n}$ for the closed half-space $\left\{\left(y_{1}, \ldots, y_{n}\right) \in \mathbb{R}^{n} \mid y_{n} \geq 0\right\}$. Fix a real number $I \in(0,1)$ and an integer number $q>n+2$. We will encounter the classical Sobolev-type spaces $W_{q}^{2,1}\left(\mathbb{R}^{n} \times(0, I)\right)$ and $W_{q}^{2,1}\left(\mathbb{R}_{+, 0}^{n} \times(0, I)\right)$ of real-values functions. Their precise definitions can be found in several sources such as, for example, 15 , Chapter I]. Given a domain $\Theta$ in $\mathbb{R}^{n}$ or $\mathbb{R}_{+}^{n}$ and a number $\lambda>0$, we will deal with the Hölder-type space $H^{\lambda, \frac{\lambda}{2}}(\Theta \times(0, I))$ of real-valued functions. Again, one may find its definition in [15, Chapter I].

Like in the beginning of Section 2, consider a vector bundle $\mathfrak{E}=\mathfrak{E}_{1} \otimes \cdots \otimes \mathfrak{E}_{k}$. Let $C^{\infty}(M \times[0, I] \rightarrow \mathfrak{E})$ be the set of all the smooth mappings $\phi: M \times[0, I] \rightarrow \mathfrak{E}$ such that the projection of $\phi(x, t)$ onto $M$ always equals $x$. Suppose $d x$ and $d t$ are the Riemannian volume measure on $M$ and the Lebesgue measure on $[0, I]$. We will encounter the space $L^{q}(M \times[0, I] \rightarrow \mathfrak{E}, d x d t)$. It is the completion of $C^{\infty}(M \times[0, I] \rightarrow \mathfrak{E})$ in the norm

$$
\|\phi\|_{L^{q}(M \times[0, I] \rightarrow \mathfrak{E}, d x d t)}=\left(\int_{M \times[0, I]}|\phi|^{q} d x d t\right)^{\frac{1}{q}} .
$$

Our further arguments will involve the spaces $L^{q}(M \times[0, I] \rightarrow \mathfrak{E}, d x d t)$ for several different bundles $\mathfrak{E}$. It will be convenient to use the same short notation $L_{I}^{q}$ for all these spaces. The norms $\|\cdot\|_{L^{q}(M \times[0, I] \rightarrow \mathfrak{E}, d x d t)}$ will all be written as $\|\cdot\|_{L_{I}^{q}}$.

Suppose $C_{W}^{\infty}(M \times[0, I] \rightarrow E)$ is the set of all the smooth mappings $\phi \in C^{\infty}(M \times[0, I] \rightarrow E)$ such that the equalities

$$
\begin{aligned}
\operatorname{Pr}_{W}(\phi(x, t)) & =o(x), & & x \in \partial M, t \in[0, I], \\
\phi(x, 0) & =o(x), & & x \in M,
\end{aligned}
$$

hold true. Let $\mathcal{W}_{q}^{I}$ stand for the completion of $C_{W}^{\infty}(M \times[0, I] \rightarrow E)$ in the norm

$$
\|\phi\|_{\mathcal{W}_{q}^{I}}=\|\phi\|_{L_{I}^{q}}+\|\nabla \nabla \phi\|_{L_{I}^{q}}+\left\|\frac{\partial}{\partial t} \phi\right\|_{L_{I}^{q}} .
$$

The space $\mathcal{W}_{q}^{I}$ is a Sobolev-type space. It will play an important part in our proof of Theorem 2.1.

Before proceeding, we need to introduce a family of atlases on $M$. If $x \in M$ and $r>0$, suppose $B(x, r)$ is the open ball in $M$ centered at $x$ of radius $r$. Given $s>0$, consider an atlas $\left(U_{k}^{s}, \bar{\alpha}_{s, k}\right)_{k=1}^{N(s)}$ on $M$ such that the following requirements hold:

1. The map $\bar{\alpha}_{s, k}$ is a diffeomorphism from $U_{k}^{s}$ to $\mathbb{R}^{n}$ if $U_{k}^{s}$ lies in $M^{\circ}$ and a diffeomorphism from $U_{k}^{s}$ to $\mathbb{R}_{+}^{n}$ if $U_{k}^{s}$ intersects $\partial M$.

2. For every $k=1, \ldots, N(s)$, the domain $U_{k}^{s}$ equals $B\left(x^{s, k}, s\right)$ with $x^{s, k} \in M$. The map $\bar{\alpha}_{s, k}$ takes $x^{s, k}$ to the origin.

3. Given $x \in M$, there exists $k$ such that $x \in B\left(x^{s, k}, \frac{s}{2}\right)$ and the distance from $x$ to $M \backslash B\left(x^{s, k}, \frac{s}{2}\right)$ is greater than $\tilde{s} s$. This requirement must hold for some number $\tilde{s}>0$ independent of $s$.

4. If $1 \leq k_{1}<\cdots<k_{N_{0}} \leq N(s)$, then $U_{k_{1}}^{s} \cap \cdots \cap U_{k_{N_{0}}}^{s}=\emptyset$. This must hold for some natural number $N_{0}$ independent of $s$.

5. The inequality

$$
\frac{1}{c^{\prime}}\left|d \bar{\alpha}_{s, k}(\xi)\right|_{\mathbb{R}^{n}} \leq|\xi| \leq c^{\prime}\left|d \bar{\alpha}_{s, k}(\xi)\right|_{\mathbb{R}^{n}}
$$

is satisfied for all $\xi$ tangent to $M$ at a point $x$ whenever $x \in B\left(x^{s, k}, \frac{3 s}{4}\right)$. Here, $|\cdot|_{\mathbb{R}_{n}}$ is the standard Euclidean norm in $\mathbb{R}^{n}$, and $c^{\prime}>0$ is a constant independent of $s, k$, and $x$. 
It is clear that $\left(U_{k}^{s}, \bar{\alpha}_{s, k}\right)_{k=1}^{N(s)}$ exists as long as $s$ is sufficiently small; cf. [15, page 295]. In what follows, we fix $s_{0}>0$ such that we can construct $\left(U_{k}^{s}, \bar{\alpha}_{s, k}\right)_{k=1}^{N(s)}$ when $s \in\left(0, s_{0}\right]$. It will be convenient for us to extend the diffeomorphisms $\bar{\alpha}_{s, k}$ to the sets $U_{k}^{s} \times[0, I]$. More precisely, we introduce the mappings $\alpha_{s, k}$ on $U_{k}^{s} \times[0, I]$ by letting $\alpha_{s, k}(x, t)=\left(\bar{\alpha}_{s, k}(x), t\right)$.

Let us define a few cut-off functions. For each $s \in\left(0, s_{0}\right]$ and $k=1, \ldots, N(s)$, choose a smooth $\bar{\kappa}_{s, k}$ : $M \rightarrow[0,1]$ identically equal to 1 on $B\left(x^{s, k}, \frac{s}{2}\right)$ and to 0 on $M \backslash B\left(x^{s, k}, \frac{3 s}{4}\right)$. We may assume the norm of the gradient of $\bar{\kappa}_{s, k}$ is bounded by $\frac{c^{\prime \prime}}{s}$ on $M$, while the norm of the Hessian of $\bar{\kappa}_{s, k}$ is bounded by $\frac{c^{\prime \prime}}{s^{2}}$ for some constant $c^{\prime \prime}>0$ independent of $s$ and $k$. It will be convenient for us to define

$$
\bar{\rho}_{s, k}=\frac{\bar{\kappa}_{s, k}}{\sum_{k=1}^{N(s)}\left(\bar{\kappa}_{s, k}\right)^{2}} .
$$

Finally, we introduce the function $\kappa_{s, k}$ on $M \times[0, I]$ by setting $\kappa_{s, k}(x, t)=\bar{\kappa}_{s, k}(x)$.

Assume that, for every $s \in\left(0, s_{0}\right]$ and $k=1, \ldots, N(s)$, there is a local trivialization $\bar{\beta}_{s, k}: \pi^{-1}\left(U_{k}^{s}\right) \rightarrow$ $U_{k}^{s} \times \mathbb{R}^{d}$ of the bundle $E$. This does not lead to any loss of generality. Along with $\bar{\beta}_{s, k}$, let us introduce a mapping $\beta_{s, k}: \pi^{-1}\left(U_{k}^{s}\right) \rightarrow \mathbb{R}^{d}$. By definition, the image of $\theta \in \pi^{-1}\left(U_{k}^{s}\right)$ under $\beta_{s, k}$ is the projection of $\bar{\beta}_{s, k}(\theta)$ onto $\mathbb{R}^{d}$. We assume that the standard Euclidean norm of $\beta_{s, k}(\theta)$ equals $|\theta|$ for all $\theta \in \pi^{-1}\left(U_{k}^{s}\right)$ and, if $U_{k}^{s}$ intersects $\partial M$, the equality

$$
\beta_{s, k}\left(\pi^{-1}(x) \cap W\right)=\left\{\left(e_{1}, \ldots, e_{d^{\prime}}, 0, \ldots, 0\right) \in \mathbb{R}^{d} \mid e_{1}, \ldots, e_{d^{\prime}} \in \mathbb{R}\right\}, \quad x \in U_{k}^{s} \cap \partial M,
$$

holds for some $d^{\prime}$ between 0 and $d$. Again, these assumptions do not lead to any loss of generality.

Let $\left(U_{k_{l}}^{s_{0}}\right)_{l=1}^{N_{1}}$ be the collection of all those $U_{k}^{s_{0}}$ that intersect $\partial M$. We use the notation $V_{l}$ for $U_{k_{l}}^{s_{0}} \cap \partial M$. It will be convenient for us to write $\hat{\alpha}_{l}$ and $\hat{\kappa}_{l}$ for the restrictions of $\bar{\alpha}_{s_{0}, k_{l}}$ and $\bar{\kappa}_{s_{0}, k_{l}}$ to $V_{l}$. One may view $\hat{\alpha}_{l}$ as a diffeomorphism from $V_{l}$ to $\mathbb{R}^{n-1}$. We can extend $\hat{\alpha}_{l}$ and $\hat{\kappa}_{l}$ to the maps $\check{\alpha}_{l}$ and $\check{\kappa}_{l}$ on $V_{l} \times[0, I]$ by setting $\check{\alpha}_{l}(x, t)=\left(\hat{\alpha}_{l}(x), t\right)$ and $\check{\kappa}_{l}(x, t)=\hat{\kappa}_{l}(x)$. Also, let $\breve{\beta}_{l}$ be the restriction of $\beta_{s_{0}, k_{l}}$ to $\pi^{-1}\left(V_{l}\right)$.

Denote $\delta=1-\frac{1}{q}$, where $q$ is the integer fixed above. We consider the fractional-order Sobolev-type space $W_{q}^{\delta, \frac{\delta}{2}}\left(\mathbb{R}^{n-1} \times(0, I)\right)$ of real-valued functions. Its precise definition may be found in [15, Chapter II]. Given a mapping $\phi: \partial M \times[0, I] \rightarrow W^{\perp}$ such that $\pi(\phi(x, t))=x$, let us write $\phi_{l}^{m}$ for the function taking $(y, t) \in \mathbb{R}^{n-1} \times[0, I]$ to the $m$ th component of the vector $\left(\breve{\beta}_{l} \circ \check{\kappa}_{l} \phi \circ \check{\alpha}_{l}^{-1}\right)(y, t) \in \mathbb{R}^{d}$. Here, $l$ is an integer between 1 and $N_{1}$, while $m$ is an integer between 1 and $d$. We introduce the space $W_{q}^{\delta, \frac{\delta}{2}}\left(\partial M \times[0, I] \rightarrow W^{\perp}\right)$. It consists of the mappings $\phi: \partial M \times[0, I] \rightarrow W^{\perp}$ such that $\pi(\phi(x, t))=x$ for all $x \in \partial M$ and the function $\phi_{l}^{m}$ lies in $W_{q}^{\delta, \frac{\delta}{2}}\left(\mathbb{R}^{n-1} \times(0, I)\right)$ for all $l=1, \ldots, N_{1}$ and $m=1, \ldots, d$. The norm of $\phi$ in $W_{q}^{\delta, \frac{\delta}{2}}\left(\partial M \times[0, I] \rightarrow W^{\perp}\right)$ is defined as the sum of the norms of $\phi_{l}^{m}$ in $W_{q}^{\delta, \frac{\delta}{2}}\left(\mathbb{R}^{n-1} \times(0, I)\right)$. We use the notation $\|\phi\|_{W_{q, I}^{\delta, \frac{\delta}{2}}}$ for it. Let us also introduce the space $\mathcal{W}_{q, I}^{\delta, \frac{\delta}{2}}$ of those $\phi \in W_{q}^{\delta, \frac{\delta}{2}}\left(\partial M \times[0, I] \rightarrow W^{\perp}\right)$ that satisfy $\phi(x, 0)=o(x)$ for all $x \in \partial M$. Clearly, $\mathcal{W}_{q, I}^{\delta, \frac{\delta}{2}}$ inherits the norm $\|\cdot\|_{W_{q, I}^{\delta, \frac{\delta}{2}}}$ from $W_{q}^{\delta, \frac{\delta}{2}}\left(\partial M \times[0, I] \rightarrow W^{\perp}\right)$. The nature of $\mathcal{W}_{q, I}^{\delta, \frac{\delta}{2}}$ is explained in part by the discussion on page 312 of [15]. Roughly speaking, this space consists of the normal derivatives of the mappings from $\mathcal{W}_{q}^{I}$.

\subsection{Linear parabolic equations}

In order to prove Theorem 2.1, we need to establish a few facts about second-order linear parabolic equations for sections of $E$. We will heavily use material from [15. Let us lay down our setup.

Consider a smooth mapping

$$
K: M \times[0, I] \rightarrow T M \otimes T M .
$$

Assume that $K(x, t)$ is a symmetric tensor over $x$ for all $x$ and $t$. One may view $K$ as a section of the bundle $T M \otimes T M$ depending on $t \in[0, I]$. Consider one more mapping

$$
G: M \times[0, I] \rightarrow E .
$$


We demand that $G \in L_{I}^{q}$. Our interest is in the equation

$$
v_{t}(x, t)-K^{i j}(x, t) \nabla_{i} \nabla_{j} v(x, t)=G(x, t), \quad x \in M^{\circ}, t \in(0, I) .
$$

The unknown $v$ is a section of $E$ dependent on $t \in[0, I)$. The subscript $t$ designates the differentiation in $t \in(0, I)$.

Consider yet another mapping

$$
p: \partial M \times[0, I] \rightarrow W^{\perp} .
$$

We suppose it lies in $\mathcal{W}_{q, I}^{\delta, \frac{\delta}{2}}$. Let us supplement (2.7) with the boundary condition

$$
\operatorname{Pr}_{W^{\perp}}\left(K^{i j}(x, t) \nu_{i}(x) \nabla_{j} v(x, t)\right)=p(x, t), \quad x \in \partial M, t \in(0, I) .
$$

Also, we assume there is a constant $c_{2}>0$ such that

$$
K^{i j}(x, t) \xi_{i} \xi_{j} \geq c_{2}|\xi|^{2}
$$

for every $x \in M, t \in[0, I]$, and $\xi \in T^{*} M$ projecting on $x$. It is now time to state the main result of this subsection. It gives us a solution to problem (2.7)-(2.8) in the space $\mathcal{W}_{q}^{I}$ as well as an important estimate. The proof will be largely based on the arguments in [15, Chapter IV]; see also [27, and [15, Chapter VII].

Lemma 2.6. The boundary value problem (2.7)-(2.8), subject to condition (2.9), has a unique solution $v$ in the space $\mathcal{W}_{q}^{I}$. Furthermore, there exists a $>0$ such that $v$ satisfies the estimate

$$
\|v\|_{\mathcal{W}_{q}^{I}} \leq a\left(\|G\|_{L_{I}^{q}}+\|p\|_{W_{q, I}^{\delta, \frac{\delta}{2}}}\right) .
$$

Remark 2.7. The number $a>0$ can be chosen independent of $I \in(0,1)$. Just how large it has to be is determined by, among other things, the mapping $K$.

Proof of Lemma 2.6. We will produce the solution $v$ and establish (2.10) assuming the number $I \in(0,1)$ is less than some number $I_{0}>0$ to be specified later. In the end of the proof, we will remove this assumption. Meanwhile, let $\mathfrak{H}_{q}^{I}$ stand for the direct sum $L_{I}^{q} \oplus \mathcal{W}_{q, I}^{\delta, \frac{\delta}{2}}$. Define the operator $\mathcal{A}: \mathcal{W}_{q}^{I} \rightarrow \mathfrak{H}_{q}^{I}$ by setting $\mathcal{A} w=\left(\mathcal{A}_{1} w, \mathcal{A}_{2} w\right)$ with

$$
\begin{aligned}
& \left(\mathcal{A}_{1} w\right)(x, t)=w_{t}(x, t)-K^{i j}(x, t) \nabla_{i} \nabla_{j} w(x, t), \quad x \in M^{\circ}, t \in(0, I), \\
& \left(\mathcal{A}_{2} w\right)(x, t)=\operatorname{Pr}_{W^{\perp}}\left(K^{i j}(x, t) \nu_{i}(x) \nabla_{j} w(x, t)\right), \quad x \in \partial M, t \in(0, I) .
\end{aligned}
$$

We need to show that $\mathcal{A}$ has a bounded inverse $\mathcal{A}^{-1}$. The assertions of the lemma will follow immediately. The role of the constant $a>0$ in inequality (2.10) will be played by the norm of $\mathcal{A}^{-1}$.

To demonstrate that $\mathcal{A}$ has a bounded inverse, we blend the arguments from the proofs of Theorems 5.3 and 9.1 in [15, Chapter IV]. Note that the geometric nature of problem (2.7)-(2.8) forces us to modify those arguments rather substantially. Our first step is to construct, assuming $I$ is less than $I_{0}$, a bounded operator $\mathcal{B}: \mathfrak{H}_{q}^{I} \rightarrow \mathcal{W}_{q}^{I}$ such that the norms of the operators $\mathcal{A B}-\operatorname{Id}_{\mathfrak{H}_{q}^{I}}$ and $\mathcal{B} \mathcal{A}-\operatorname{Id}_{\mathcal{W}_{q}^{I}}$ are less than 1. Here, $\operatorname{Id}_{\mathfrak{H}_{q}^{I}}$ and $\operatorname{Id}_{\mathcal{W}_{q}^{I}}$ are the identity maps in the corresponding spaces. Once $\mathcal{B}$ is at hand, we will utilize it to produce a left inverse and a right inverse for $\mathcal{A}$. The existence of $\mathcal{A}^{-1}$ will be a direct consequence.

Suppose $\left(J_{1}, J_{2}\right) \in \mathfrak{H}_{q}^{I}$. In order to specify how the operator $\mathcal{B}$ acts on $\left(J_{1}, J_{2}\right)$, let us fix $s \in\left(0, s_{0}\right]$ and an atlas $\left(U_{k}^{s}, \bar{\alpha}_{s, k}\right)_{k=1}^{N(s)}$ on $M$ as described in Section 2.2. Along with $\left(U_{k}^{s}, \bar{\alpha}_{s, k}\right)_{k=1}^{N(s)}$, we have the collection $\left(\beta_{s, k}\right)_{k=1}^{N(s)}$. Each $\beta_{s, k}$ is a mapping from $\pi^{-1}\left(U_{k}^{s}\right)$ to $\mathbb{R}^{d}$. Choose a domain $U_{k}^{s}$ intersecting $\partial M$. The diffeomorphism $\bar{\alpha}_{s, k}$ takes $U_{k}^{s}$ to $\mathbb{R}_{+}^{n}$. Let $\left\{y_{1}, \ldots, y_{n}\right\}$ be the standard coordinates on $\mathbb{R}_{+}^{n}$. Given $y \in \mathbb{R}_{+}^{n}$ and $t \in[0, I]$, we write $\hat{K}_{s, k}^{i j}(y, t)$ and $\hat{\nu}_{i}^{s, k}(y)$ for the components of the tensors $K\left(\bar{\alpha}_{s, k}^{-1}(y), t\right)$ and $\nu\left(\bar{\alpha}_{s, k}^{-1}(y)\right)$ with respect to $\bar{\alpha}_{s, k}$ and $\left\{y_{1}, \ldots, y_{n}\right\}$. 
To describe the action of $\mathcal{B}$ on $\left(J_{1}, J_{2}\right)$, some preparations are required. It will be convenient for us to denote

$$
\begin{array}{ll}
\hat{J}_{1, s, k}(y, t)=\left(\beta_{s, k} \circ \kappa_{s, k} J_{1} \circ \alpha_{s, k}^{-1}\right)(y, t), & y \in \mathbb{R}_{+}^{n}, t \in(0, I), \\
\hat{J}_{2, s, k}(y, t)=\left(\beta_{s, k} \circ \kappa_{s, k} J_{2} \circ \alpha_{s, k}^{-1}\right)(y, t), & y \in \partial \mathbb{R}_{+}^{n}, t \in(0, I) .
\end{array}
$$

Consider the equation

$$
z_{t}(y, t)-\hat{K}_{s, k}^{i j}(0,0) z_{y_{i} y_{j}}(y, t)=\hat{J}_{1, s, k}(y, t), \quad y \in \mathbb{R}_{+, 0}^{n}, t \in(0, I) .
$$

Here, $z=\left(z^{1}, \ldots, z^{d}\right)$ is the vector of unknown functions with $z^{m}: \mathbb{R}_{+}^{n} \times[0, I) \rightarrow \mathbb{R}$ for $m=1, \ldots, d$. The subscript $y_{i}$ and $y_{j}$ mean component-wise differentiation in $y_{i}$ and $y_{j}$. Let us impose the boundary conditions

$$
\begin{aligned}
\operatorname{Pr}_{\beta_{s, k}\left(\pi^{-1}\left(\bar{\alpha}_{s, k}^{-1}(y)\right) \cap W\right)} z(y, t) & =0, \\
\operatorname{Pr}_{\beta_{s, k}\left(\pi^{-1}\left(\bar{\alpha}_{s, k}^{-1}(y)\right) \cap W^{\perp}\right)}\left(\hat{K}_{s, k}^{i j}(0,0) \hat{\nu}_{i}^{s, k}(y) z_{y_{j}}(y, t)\right) & =\hat{J}_{2, s, k}(y, t), \quad y \in \partial \mathbb{R}_{+}^{n}, t \in(0, I),
\end{aligned}
$$

and the initial condition

$$
z(y, 0)=0, \quad y \in \mathbb{R}_{+}^{n} .
$$

Using formula (2.6), we see that problem (2.11)-(2.12)-(2.13) is equivalent to $d$ uncoupled problems, one for each $z^{m}$. The Dirichlet boundary condition is imposed on $z^{m}$ when $m=1, \ldots, d^{\prime}$, and a Neumann-type condition is in place when $m=d^{\prime}+1, \ldots, d$. On the basis of Theorem 6.1 from [15, Chapter IV] (see also the argument on pages 343-345 of [15]), one easily concludes that problem (2.11)-(2.12)-(2.13) has a unique solution whose components all lie in the space $W_{q}^{2,1}\left(\mathbb{R}_{+, 0}^{n} \times(0, I)\right)$. We denote this solution by $\hat{\mathcal{B}}_{J_{1}, J_{2}}^{s, k}$. Given $m=1, \ldots, d$, let $\hat{J}_{1, s, k}^{m}, \hat{J}_{2, s, k}^{m}$, and $\hat{\mathcal{B}}_{J_{1}, J_{2}}^{s, k, m}$ be the $m$ th components of $\hat{J}_{1, s, k}, \hat{J}_{2, s, k}$, and $\hat{\mathcal{B}}_{J_{1}, J_{2}}^{s, k}$. The estimate

$$
\left\|\hat{\mathcal{B}}_{J_{1}, J_{2}}^{s, k, m}\right\|_{W_{q}^{2,1}\left(\mathbb{R}_{+, 0}^{n} \times(0, I)\right)} \leq a_{1}\left(\left(\int_{\mathbb{R}_{+}^{n} \times[0, I]}\left|\hat{J}_{1, s, k}^{m}\right|^{q} d y d t\right)^{\frac{1}{q}}+\left\|\hat{J}_{2, s, k}^{m}\right\|_{W_{q}^{\delta, \frac{\delta}{2}}\left(\mathbb{R}^{n-1} \times(0, I)\right)}\right)
$$

must be satisfied for some $a_{1}>0$ ( $d y$ and $d t$ are the Lebesgue measures on $\mathbb{R}_{+}^{n}$ and $\left.[0, I]\right)$. We introduce the $\operatorname{map} \mathcal{B}_{J_{1}, J_{2}}^{s, k}: U_{k}^{s} \times[0, I] \rightarrow E$ by setting

$$
\mathcal{B}_{J_{1}, J_{2}}^{s, k}(x, t)=\bar{\beta}_{s, k}^{-1}\left(x,\left(\hat{\mathcal{B}}_{J_{1}, J_{2}}^{s, k} \circ \alpha_{s, k}\right)(x, t)\right), \quad x \in U_{k}^{s}, t \in[0, I] .
$$

It will be convenient for us to have $\mathcal{B}_{J_{1}, J_{2}}^{s, k}$ defined on all of $M \times[0, I]$. Therefore, we let $\mathcal{B}_{J_{1}, J_{2}}^{s, k}(x, t)=o(x)$ for $x \in M \backslash U_{k}^{s}$ and $t \in[0, I]$. The map $\mathcal{B}_{J_{1}, J_{2}}^{s, k}$ will be a substantial ingredient in the image of $\left(J_{1}, J_{2}\right)$ under $\mathcal{B}$.

So far, we've been assuming $U_{k}^{s}$ intersected $\partial M$. Suppose now $U_{k}^{s}$ is a domain contained in $M^{\circ}$. The diffeomorphism $\bar{\alpha}_{s, k}$ then acts from $U_{k}^{s}$ to $\mathbb{R}^{n}$. We can write down an equation analogous to (2.11) in $\mathbb{R}^{n}$ and an initial condition analogous to (2.13) in $\mathbb{R}^{n}$. The resulting problem will have a unique solution with components in $W_{q}^{2,1}\left(\mathbb{R}^{n} \times(0, I)\right)$. Again, we denote this solution by $\hat{\mathcal{B}}_{J_{1}, J_{2}}^{s, k}$ and introduce the map $\mathcal{B}_{J_{1}, J_{2}}^{s, k}$. Notice that each component of $\hat{\mathcal{B}}_{J_{1}, J_{2}}^{s, k}$ will satisfy an estimate similar to (2.14). We are now ready to specify how $\mathcal{B}$ acts on $\left(J_{1}, J_{2}\right)$.

Consider the mapping $\mathcal{B}_{J_{1}, J_{2}}^{s}: M \times[0, I] \rightarrow E$ given by the formula

$$
\mathcal{B}_{J_{1}, J_{2}}^{s}(x, t)=\sum_{k=1}^{N(s)} \bar{\rho}_{s, k}(x) \mathcal{B}_{J_{1}, J_{2}}^{s, k}(x, t), \quad x \in M, t \in[0, I]
$$

It is clear that $\mathcal{B}_{J_{1}, J_{2}}^{s} \in \mathcal{W}_{q}^{I}$. Let $\mathcal{B}$ be the operator taking $\left(J_{1}, J_{2}\right) \in \mathfrak{H}_{q}^{I}$ to $\mathcal{B}_{J_{1}, J_{2}}^{s} \in \mathcal{W}_{q}^{I}$. Our next step is to establish a few inequalities for $\mathcal{B}$.

In the beginning of the proof, we assumed $I$ was less than some $I_{0}>0$. Fix a number $\bar{s} \in(0,1]$ and suppose $I_{0}$ is $\bar{s} s^{2}$. Thus, the formula

$$
I<\bar{s} s^{2}
$$


must hold. Employing (2.14) $(2.15)$, and the properties of the atlas $\left(U_{k}^{s}, \bar{\alpha}_{s, k}\right)_{k=1}^{N(s)}$ listed in Section 2.2, we easily see that the inequality

$$
\|\mathcal{B} h\|_{\mathcal{W}_{q}^{I}}<a_{2}\|h\|_{\mathfrak{H}_{q}^{I}}, \quad h \in \mathfrak{H}_{q}^{I},
$$

is satisfied for some $a_{2}>0$ independent of the numbers $s$ and $\bar{s}$; cf. Lemma 4.7 and Theorem 7.1 in [15], Chapter IV]. In particular, the operator $\mathcal{B}$ is bounded.

If $s$ and $\bar{s}$ are chosen sufficiently small and (2.15) holds, then

$$
\begin{aligned}
\|\mathcal{A B} h-h\|_{\mathfrak{H}_{q}^{I}}<\|h\|_{\mathfrak{H}_{q}^{I}}, \quad h \in \mathfrak{H}_{q}^{I}, \\
\|\mathcal{B} \mathcal{A} w-w\|_{\mathcal{W}_{q}^{I}}<\|w\|_{\mathcal{W}_{q}^{I}}, \quad w \in \mathcal{W}_{q}^{I} .
\end{aligned}
$$

In order to prove this, it is necessary to write down a series of estimates based on (2.16) and the Hölder inequality. These estimates are very similar to the ones on pages 348-349 of [15]; see also [27. We will not present them here. At this point, the required inequalities for $\mathcal{B}$ are at hand. We will now utilize $\mathcal{B}$ to produce the left inverse and the right inverse of $\mathcal{A}$.

If $s$ and $\bar{s}$ are chosen small and (2.15) holds, then the norms of the operators $\mathcal{A B}-\operatorname{Id}_{\mathfrak{H}_{q}^{I}}$ and $\mathcal{B A}-\operatorname{Id}_{\mathcal{W}_{q}^{I}}$ are less than 1. In this case, $\mathcal{A B}=\left(\mathcal{A B}-\operatorname{Id}_{\mathfrak{H}_{q}^{I}}\right)+\operatorname{Id}_{\mathfrak{H}_{q}^{I}}$ and $\mathcal{B A}=\left(\mathcal{B A}-\operatorname{Id}_{\mathcal{W}_{q}^{I}}\right)+\operatorname{Id}_{\mathcal{W}_{q}^{I}}$ must have bounded inverses. Keeping this in mind, we conclude that

$$
\begin{aligned}
\mathcal{A}\left(\mathcal{B}(\mathcal{A B})^{-1}\right) & =\operatorname{Id}_{\mathfrak{H}_{q}^{I}}, \\
\left((\mathcal{B A})^{-1} \mathcal{B}\right) \mathcal{A} & =\operatorname{Id}_{\mathcal{W}_{q}^{I}} .
\end{aligned}
$$

It is now easy to see that $\mathcal{A}$ must have a bounded inverse $\mathcal{A}^{-1}$. Thus, the assertions of the lemma hold true provided $I$ is less than $\bar{s} s^{2}$ for some $s$ and $\bar{s}$. In order to complete the proof, we have to remove the assumption on $I$. But this can be accomplished by repeating the arguments from pages 349-350 of [15] (see also [15, Chapter IV, Section 8]).

\subsection{Proof of the existence theorem}

Our preparations for the proof of Theorem 2.1 are now completed. We proceed in two steps. First, we will use a fixed-point argument similar to the one found in [30] (see also [12, 1]) to construct a solution $u$ of problem (2.1)-(2.2)-(2.3). Then we will employ classical facts from [15] to show that $u$ possesses the desired differentiability properties.

Proof of Theorem 2.1. Let us assume $u_{0}(x)$ is equal to zero for every $x \in M$. This does not lead to any loss of generality. Indeed, it is always possible to reduce the general case to the case where $u_{0}(x)=o(x)$ for all $x \in M$ by introducing the new unknown $\check{u}(x, t)=u(x, t)-u_{0}(x)$.

We will now construct the mapping $\mathcal{C}$ whose fixed point will be a solution of problem (2.1)-(2.2)-(2.3). As in Sections 2.2 and 2.3. suppose $I \in(0,1)$ is a real number and $q>n+2$ is an integer. The space $\mathcal{W}_{q}^{I}$ will play an important role in our further considerations. Denote $H_{0}(x)=H(o(x), 0)$ for each $x \in M$. Suppose that $w \in \mathcal{W}_{q}^{I}$. Let us introduce the mapping $H_{w}: M \times[0, I] \rightarrow T M \otimes T M$ by the formula

$$
H_{w}(x, t)=H(w(x, t), t), \quad x \in M, t \in[0, I] .
$$

The notation

$$
\begin{aligned}
F_{w, \nabla w}(x, t) & =F(w(x, t), \nabla w(x, t), t), \quad x \in M, t \in[0, I], \\
\Psi_{w}(x, t) & =\Psi(w(x, t), t), \quad x \in \partial M, t \in[0, I],
\end{aligned}
$$

will also be helpful. We consider the equation

$$
\begin{aligned}
v_{t}(x, t) & -H_{0}^{i j}(x) \nabla_{i} \nabla_{j} v(x, t) \\
& =F_{w, \nabla w}(x, t)+\left(H_{w}^{i j}(x, t)-H_{0}^{i j}(x)\right) \nabla_{i} \nabla_{j} w(x, t), \quad x \in M^{\circ}, t \in(0, I),
\end{aligned}
$$


for the unknown section $v$ depending on $t \in[0, I]$. We then supplement this equation with the boundary condition

$$
\begin{aligned}
\operatorname{Pr}_{W^{\perp}} & \left(H_{0}^{i j}(x) \nu_{i}(x) \nabla_{j} v(x, t)\right) \\
& =\Psi_{w}(x, t)-\operatorname{Pr}_{W^{\perp}}\left(\left(H_{w}^{i j}(x, t)-H_{0}^{i j}(x)\right) \nu_{i}(x) \nabla_{j} w(x, t)\right), \quad x \in \partial M, t \in(0, I) .
\end{aligned}
$$

Lemma 2.6 above demonstrates that problem (2.17)-(2.18) has a unique solution in the space $\mathcal{W}_{q}^{I}$. We may, therefore, define a mapping $\mathcal{C}: \mathcal{W}_{q}^{I} \rightarrow \mathcal{W}_{q}^{I}$ that takes $w$ to this solution. A series of estimates based on (2.10) show the existence of a number $T \in(0,1)$ such that $\mathcal{C}$ has a fixed point when $I \leq T$. These estimates are very similar to the ones in the proofs of Lemmas 2.4 and 2.5 in 30 . We will not present them here. It is, thus, possible to find $w \in \mathcal{W}_{q}^{I}$ satisfying the equality $\mathcal{C}(w)=w$ provided $I \leq T$. In particular, there exists $u \in \mathcal{W}_{q}^{T}$ with $\mathcal{C}(u)=u$. Lemma 3.3 of [15, Chapter II] implies that $u$ and $\nabla u$ are continuous on $M \times[0, T)$. Formulas (2.1) and (2.2) hold for $u$. We also have (2.3) since we assumed $u_{0}(x)=o(x)$ for $x \in M$. It remains to show that $u$ is smooth on $M \times(0, T)$. We will do so on the basis of bootstrapping argument.

Fix an atlas $\left(U_{k}^{s}, \bar{\alpha}_{s, k}\right)_{k=1}^{N(s)}$ on $M$ as described in Section 2.2. Here, $s$ is an arbitrary positive number less than $s_{0}$. Along with $\left(U_{k}^{s}, \bar{\alpha}_{s, k}\right)_{k=1}^{N(s)}$, we have the collection $\left(\beta_{s, k}\right)_{k=1}^{N(s)}$. Choose a domain $U_{k}^{s}$ intersecting $\partial M$. The diffeomorphism $\bar{\alpha}_{s, k}$ takes $U_{k}^{s}$ to $\mathbb{R}_{+}^{n}$. We introduce the function $\tilde{u}_{s, k}=\beta_{s, k} \circ u \circ \alpha_{s, k}^{-1}$. It acts from $\mathbb{R}_{+}^{n} \times[0, T]$ to $\mathbb{R}^{d}$. We take $m=1, \ldots, d$ and write $\tilde{u}_{s, k}^{m}$ for the $m$ th component of $\tilde{u}_{s, k}$. Our next step is to study the differentiability of $\tilde{u}_{s, k}^{m}$. This will help us obtain the desired conclusion about the smoothness of $u$.

Let $\left\{y_{1}, \ldots, y_{n}\right\}$ be the standard coordinates in $\mathbb{R}_{+}^{n}$. In what follows, the notation $D \tilde{u}_{s, k}$ stands for the Jacobian matrix of $\tilde{u}_{s, k}$ with respect to $\left\{y_{1}, \ldots, y_{n}\right\}$. It is not difficult to understand on the basis of (2.1) that $\tilde{u}_{s, k}^{m}$ satisfies

$$
\left(\tilde{u}_{s, k}^{m}\right)_{t}(y, t)-\tilde{H}_{s, k, u}^{i j}(y, t)\left(\tilde{u}_{s, k}^{m}\right)_{y_{i} y_{j}}(y, t)=\tilde{F}_{u, \nabla u}^{s, k, m}(y, t), \quad y \in \mathbb{R}_{+, 0}^{n}, t \in(0, T) .
$$

In this formula,

$$
\tilde{H}_{s, k, u}^{i j}(y, t)=\tilde{H}_{s, k}^{i j}\left(\tilde{u}_{s, k}(y, t), y, t\right), \quad y \in \mathbb{R}_{+, 0}^{n}, t \in(0, T),
$$

with $\tilde{H}_{s, k}^{i j}$ being a function from $\mathbb{R}^{d} \times \mathbb{R}_{+}^{n} \times[0, T]$ to $\mathbb{R}$ for any $i, j=1, \ldots, n$. We point out that $\tilde{H}_{s, k}^{i j}$ is smooth in all three variables. Also, in formula (2.19),

$$
\tilde{F}_{u, \nabla u}^{s, k, m}(y, t)=\tilde{F}_{s, k}^{m}\left(\tilde{u}_{s, k}(y, t), D \tilde{u}_{s, k}(y, t), y, t\right), \quad y \in \mathbb{R}_{+, 0}^{n}, t \in(0, T),
$$

with $\tilde{F}_{s, k}^{m}$ taking $\mathbb{R}^{d} \times \mathrm{Mat}_{d, n} \times \mathbb{R}_{+}^{n} \times[0, T]$ to $\mathbb{R}$. The notation Mat ${ }_{d, n}$ refers to the space of $d \times n$ matrices. We naturally identify Mat $\mathrm{M}_{d, n}$ with $\mathbb{R}^{d n}$ and equip it with the standard Euclidean metric. Then the function $\tilde{F}_{s, k}^{m}$ is smooth in all four variables. According to (2.2) and (2.6), if $m$ is between 1 and $d^{\prime}$, the equality

$$
\tilde{u}_{s, k}^{m}(y, t)=0, \quad y \in \partial \mathbb{R}_{+}^{n}, t \in(0, T),
$$

holds true. For the other values of $m$, we have a slightly more complicated identity. Finally, it is easy to see from (2.3) that

$$
\tilde{u}_{s, k}^{m}(y, 0)=0, \quad y \in \mathbb{R}_{+}^{n} .
$$

We proceed to establishing differentiability properties of $\tilde{u}_{s, k}^{m}$.

The fact that $u$ lies in $\mathcal{W}_{q}^{T}$ and Lemma 3.3 from [15, Chapter II] tell us that the coefficients $\tilde{H}_{s, k, u}^{i j}$ and the term $\tilde{F}_{u, \nabla u}^{s, k, m}$ in equation (2.19) lie in the Hölder-type space $H^{\lambda, \frac{\lambda}{2}}\left(\Theta_{s, k} \times(0, T)\right)$ for $\Theta_{s, k}=\bar{\alpha}_{s, k}\left(B\left(x^{s, k}, \frac{s}{2}\right)\right)$ and some $\lambda \in(0,1)$. Taking (2.19)-(2.20)-(2.21) into account and employing the material in 15, Chapter III, Section 12], we can conclude that $\tilde{u}_{s, k}^{m}$ must belong to $H^{2+\lambda, 1+\frac{\lambda}{2}}\left(\Theta_{s, k} \times(0, T)\right)$ if $m=1, \ldots, d^{\prime}$. Analogous reasoning works when $m=d^{\prime}+1, \ldots, d$. In this case, $\tilde{u}_{s, k}^{m}$ is a solution of (2.19) under a Neumann-type boundary condition and the initial condition (2.21). We easily see that $\tilde{u}_{s, k}^{m} \in H^{2+\lambda, 1+\frac{\lambda}{2}}\left(\Theta_{s, k} \times(0, T)\right)$.

Let us examine equation (2.19) again. It is now evident that the coefficients $\tilde{H}_{s, k, u}^{i j}$ and the term $\tilde{F}_{u, \nabla u}^{s, k, m}$ in it must belong to $H^{1+\lambda, \frac{1}{2}+\frac{\lambda}{2}}\left(\Theta_{s, k} \times(0, T)\right)$. As above, we conclude $\tilde{u}_{s, k}^{m} \in H^{3+\lambda, \frac{3}{2}+\frac{\lambda}{2}}\left(\Theta_{s, k} \times(0, T)\right)$. Let us iterate this argument. It becomes clear that $\tilde{u}_{s, k}^{m}$ must be smooth on $\Theta_{s, k} \times(0, T)$. 
So far, it's been assumed that the domain $U_{k}^{s}$ intersected $\partial M$. Suppose now $U_{k}^{s}$ is contained in $M^{\circ}$. We can still introduce the function $\tilde{u}_{s, k}=\beta_{s, k} \circ u \circ \alpha_{s, k}^{-1}$. It now acts from $\mathbb{R}^{n} \times[0, T]$ to $\mathbb{R}^{d}$. Repeating the above reasoning with minor modifications, we can demonstrate that, given $m=1, \ldots, d$, the component $\tilde{u}_{s, k}^{m}$ must be smooth on $\bar{\alpha}_{s, k}\left(B\left(x^{s, k}, \frac{s}{2}\right)\right) \times(0, T)$.

Let us summarize. Our goal was to establish the differentiability properties of $u$ on $M \times(0, T)$. The presented arguments suggest that, whichever $k=1, \ldots, N(s)$ we choose, $u$ must be smooth on $B\left(x^{s, k}, \frac{s}{2}\right) \times$ $(0, T)$. This immediately implies the desired properties of $u$ on $M \times(0, T)$.

\section{The Ricci flow}

Like in Section 2, we consider a smooth manifold $M$ with boundary. We now assume that $M$ is $n$-dimensional with $n \geq 3$, compact, connected, and oriented. The notations $M^{\circ}$ and $\partial M$ will be used for the interior and the boundary of $M$. Our goal is to investigate the Ricci flow on $M$. More specifically, we will propose a new boundary condition and establish two short-time existence results for this flow. The proofs will be based on the method commonly known as DeTurck's trick. The reader should consult, e.g., 8, 9, 29 for a detailed explanation of this method in the context of closed manifolds. A relevant historic discussion may be found in [6. Our proofs will rely heavily on Theorem 2.1.

We focus on the equation

$$
\frac{\partial}{\partial t} g(x, t)=-2 \operatorname{Ric}^{g}(x, t), \quad x \in M^{\circ}, t \in(0, T),
$$

for a Riemannian metric $g$ on $M$ depending on the parameter $t \in[0, T)$ with $T>0$. The notation $\operatorname{Ric}^{g}$ in the right-hand side refers to the Ricci curvature of $g$. We fix a smooth Riemannian metric $\hat{g}$ on $M$ and supplement (3.1) with the initial condition

$$
g(x, 0)=\hat{g}(x), \quad x \in M .
$$

So far, we do not concern ourselves with the behavior of $g$ near $\partial M$. The reader will recognize that (3.1) is the Ricci flow equation on $M$. The introduction to the present paper contains references to several books that discuss it in great detail.

We call a mapping $g: M \times[0, T) \rightarrow T^{*} M \otimes T^{*} M$ a decent solution of problem (3.1)-(3.2) on $M \times[0, T)$ if the following requirements are met:

1. For every $x \in M$ and $t \in[0, T)$, the tensor $g(x, t)$ is symmetric and positive-definite. In other words, $g$ is a Riemannian metric on $M$ depending on $t \in[0, T)$.

2. The mapping $g$ is continuous on $M \times[0, T)$ and smooth on $M \times(0, T)$.

3. The Ricci flow equation (3.1) and the initial condition (3.2) hold for $g$.

Throughout Section 3 , we write $\tilde{\nabla}$ and $\hat{\nabla}$ for the Levi-Civita connections of the metrics $g$ and $\hat{g}$. In a similar fashion, $\tilde{v}$ and $\hat{v}$ will stand for the outward unit normal vector fields on $\partial M$ with respect to $g$ and $\hat{g}$. We point out that $\tilde{\nabla}$ and $\tilde{v}$ depend on the parameter $t \in[0, T)$, while $\hat{\nabla}$ and $\hat{v}$ do not. The connection $\hat{\nabla}$ gives rise to connections in tensor bundles over $M$. We preserve the notation $\hat{\nabla}$ for them.

As in Section 2, let us implicitly assume that a coordinate system $\left\{x_{1}, \ldots, x_{n}\right\}$ is chosen in a neighborhood of every point $x \in M$. Suppose $T$ is a $(k, l)$-tensor field on $M$ near $x$. By analogy with the notation of Section 2, we write $T_{j_{1} \ldots j_{l}}^{i_{1} \ldots i_{k}}$ for the components of $T$ in the coordinates $\left\{x_{1}, \ldots, x_{n}\right\}$, while $\tilde{\nabla}_{i} T$ and $\hat{\nabla}_{i} T$ stand for $\tilde{\nabla}_{\frac{\partial}{\partial x_{i}}} T$ and $\hat{\nabla}_{\frac{\partial}{\partial x_{i}}} T$. The expression $\hat{\nabla}_{i} \hat{\nabla}_{j} T$ means the second covariant derivative $\hat{\nabla} \hat{\nabla} T$ applied to $\frac{\partial}{\partial x_{i}}$ and $\frac{\partial}{\partial x_{j}}$. If $x$ lies in $\partial M$, we assume that $\left\{x_{1}, \ldots, x_{n-1}\right\}$ is a local coordinate system on $\partial M$, the $n$th coordinate of any point in $\partial M$ near $x$ is equal to 0 , and $\hat{v}$ is a scalar multiple of $\frac{\partial}{\partial x_{n}}$ near $x$. Given a $(k, l)$-tensor $Z$ on $\partial M$ at $x \in \partial M$, we write $Z_{\beta_{1} \ldots \beta_{l}}^{\alpha_{1} \ldots \alpha_{k}}$ for the components of $Z$ with respect to $\left\{x_{1}, \ldots, x_{n-1}\right\}$. As before, the Einstein summation convention is in effect. The Latin indices $i, j, k$, and $l$ will vary from 1 to $n$, whereas the Greek indices $\alpha, \beta, \gamma$, and $\sigma$ will vary from 1 to $n-1$.

In accordance with the notation introduced above, $g_{i j}$ and $\hat{g}_{i j}$ are the components of the Riemannian metrics $g$ and $\hat{g}$. We will also deal with the inverses of these metrics. Their components will be denoted by $g^{i j}$ and $\hat{g}^{i j}$. 


\subsection{Formulation of the existence results}

Our further considerations involve the second fundamental form field II : $\partial M \times[0, T) \rightarrow T^{*} \partial M \otimes T^{*} \partial M$ of the boundary with respect to $g$. By definition,

$$
\mathrm{II}_{\alpha \beta}(x, t)=g_{\alpha \gamma}(x, t)\left(\tilde{\nabla}_{\beta} \tilde{v}\right)^{\gamma}(x, t), \quad x \in \partial M, t \in[0, T) .
$$

Let us introduce the quantity

$$
\mathcal{H}(x, t)=\frac{1}{n-1} g^{\alpha \beta}(x, t) \mathrm{II}_{\alpha \beta}(x, t), \quad x \in \partial M, t \in[0, T) .
$$

It is called the mean curvature of $\partial M$. One may also consider the second fundamental form field of $\partial M$ with respect to $\hat{g}$. We will denote it by II. Finally, one may introduce the mean curvature of $\partial M$ with respect to $\hat{g}$. We will write $\hat{\mathcal{H}}$ for it.

Let us state the first result of this section. It assumes that $\hat{\mathcal{H}}(x)$ is independent of $x \in \partial M$. If this is the case, we can solve problem (3.1)-(3.2) for a short time maintaining control over $\mathcal{H}(x, t)$.

Theorem 3.1. Suppose the mean curvature $\hat{\mathcal{H}}(x)$ is equal to a constant $\mathcal{H}_{0} \in \mathbb{R}$ for all $x \in \partial M$. Let $\mu$ be a smooth real-valued function on $[0, \infty)$ with $\mu(0)=1$. Then there exist $T>0$ and a decent solution $g$ of problem (3.1) -(3.2) on $M \times[0, T)$ such that the mean curvature $\mathcal{H}(x, t)$ satisfies the boundary condition

$$
\mathcal{H}(x, t)=\mu(t) \mathcal{H}_{0}
$$

for all $x \in \partial M$ and $t \in[0, T)$.

Remark 3.2. We emphasize that the smooth function $\mu$ appearing in the theorem can be arbitrary as long as $\mu(0)=1$. Essentially, different choices of this function correspond to different evolutions of $\hat{g}$ under the Ricci flow. It is also reasonable to think of $\mu$ as a normalization factor. The number $T$ whose existence the theorem asserts may depend on $\mu$. The explicit form of this dependence, however, is quite difficult to track down. We refer to [4] for a discussion relevant to the geometric meaning of $\mu$.

The second result of this section touches upon the question of the behavior of the Ricci flow on manifolds with convex boundary. Again, it establishes the existence of a solution. Note that there are several ways to define what it means for $\partial M$ to be convex with respect to a Riemannian metric on $M$. Different viewpoints and the relations between them are surveyed in [21. Perhaps, the most common way is to deem $\partial M$ convex with respect to a Riemannian metric on $M$ if and only if the second fundamental form of $\partial M$ with respect to this metric is nonnegative-definite on $\partial M$. Having said that, we can formulate the result.

Proposition 3.3. There exist $T>0$, a map $\psi$ from $M \times[0, T)$ to $M$, and a decent solution $g$ of problem (3.1) - (3.2) on $M \times[0, T)$ such that the following statements hold:

1. It is the case that $\psi$ is continuous on $M \times[0, T)$ and smooth on $M \times(0, T)$.

2. The map $\psi(\cdot, t)$ is a diffeomorphism from the manifold $M$ to itself for every $t \in[0, T)$.

3. The form field $\mathrm{II}(\cdot, t)$ coincides with the pullback of II by the restriction of $\psi(\cdot, t)$ to $\partial M$ whenever $t \in$ $[0, T)$.

As a consequence, if $\hat{\mathrm{II}}(x)$ is nonnegative-definite for all $x \in \partial M$ (that is, $\partial M$ is convex with respect to $\hat{g}$ ), then $\operatorname{II}(x, t)$ is nonnegative-definite for all $x \in \partial M$ and $t \in[0, T)$ (that is, $\partial M$ remains convex with respect to $g$ ).

One more remark is in order at this point. After stating it, we will proceed to proving Theorem 3.1 and Proposition 3.3. The first step will be to make some preparations. We will do so in Section 3.2

Remark 3.4. It may be possible to improve the regularity of $g$ on the set $M \times[0, T)$ in Theorem 3.1 and Proposition 3.3 by imposing additional restrictions on the behavior of $\hat{g}$ near $\partial M$. Remark 2.4 suggests the nature of the assumptions that have to be made. We do not address this issue in the present paper. 


\subsection{The Ricci-DeTurck flow and bundles over the boundary}

Let us introduce the Ricci-DeTurck flow on the manifold $M$. We will employ it to prove Theorem 3.1 Supplementing it with boundary conditions will be the key step in our reasoning. The existence of solutions to the flow will follow from Theorem 2.1. We will then use a similar strategy to prove Proposition 3.3 .

Given a Riemannian metric $\bar{g}$ on $M$ depending on $t \in[0, T)$, define the mappings $P^{\bar{g}}: M \times[0, T) \rightarrow T^{*} M$ and $Q^{\bar{g}}: M \times[0, T) \rightarrow T^{*} M \otimes T^{*} M$ by the formulas

$$
\begin{aligned}
P_{i}^{\bar{g}}(x, t) & =\bar{g}_{i j}(x, t) \bar{g}^{k l}(x, t)\left(\bar{\Gamma}_{k l}^{j}(x, t)-\hat{\Gamma}_{k l}^{j}(x)\right), \\
Q_{i j}^{\bar{g}}(x, t) & =\left(\bar{\nabla}_{i} P^{\bar{g}}\right)_{j}(x, t)+\left(\bar{\nabla}_{j} P^{\bar{g}}\right)_{i}(x, t), \quad x \in M, t \in[0, T) .
\end{aligned}
$$

Here, $\bar{g}^{k l}$ are the components of the inverse of $\bar{g}$, while $\bar{\Gamma}_{k l}^{j}$ and $\hat{\Gamma}_{k l}^{j}$ are the Christoffel symbols corresponding to the Levi-Civita connections $\bar{\nabla}$ and $\hat{\nabla}$ of $\bar{g}$ and $\hat{g}$. We have written $\bar{\nabla}_{i} P^{\bar{g}}$ and $\bar{\nabla}_{j} P^{\bar{g}}$ for the covariant derivatives of $P^{\bar{g}}$ in the directions $\frac{\partial}{\partial x_{i}}$ and $\frac{\partial}{\partial x_{j}}$. These derivatives are taken with respect to the connection in $T^{*} M$ induced by $\bar{\nabla}$. Consider the equation

$$
\frac{\partial}{\partial t} \bar{g}(x, t)=-2 \operatorname{Ric}^{\bar{g}}(x, t)+Q^{\bar{g}}(x, t), \quad x \in M^{\circ}, t \in(0, T) .
$$

In what follows, it will be convenient for us to use the notation $\mathcal{E}$ for the bundle of symmetric $(0,2)$-tensors on $M$. One could think about $\bar{g}$ as a section of $\mathcal{E}$ depending on $t \in[0, T)$. By Lemma 7.48 in [9], we can rewrite equation (3.3) as

$$
\frac{\partial}{\partial t} \bar{g}(x, t)=\bar{g}^{i j}(x, t) \hat{\nabla}_{i} \hat{\nabla}_{j} \bar{g}(x, t)+R(\bar{g}(x, t), \hat{\nabla} \bar{g}(x, t)), \quad x \in M^{\circ}, t \in(0, T) .
$$

The map $R$ here is defined on the set of all the pairs $(\eta, \theta)$ such that $\eta$ is a symmetric positive-definite $(0,2)$ tensor and $\theta$ is a (0,3)-tensor at the same point. The values of $R$ lie in $T^{*} M \otimes T^{*} M$. Equation (3.3) is the Ricci-DeTurck flow equation on $M$. Rewriting it in the form (3.4) will later enable us to apply Theorem 2.1 to it.

Let us introduce a few more pieces of notation. Suppose $\pi_{\mathcal{E}}$ is the projection in the bundle $\mathcal{E}$. We assume that $\mathcal{E}$ is equipped with the fiber metric given by $\hat{g}$. It will be convenient for us to write $\mathcal{E}_{\partial M}$ for the set of all $\eta \in \mathcal{E}$ such that $\pi_{\mathcal{E}}(\eta) \in \partial M$. This set has the structure of a vector bundle over $\partial M$ induced by the structure of $\mathcal{E}$. It also inherits the fiber metric from $\mathcal{E}$. Let $\mathcal{F}$ be the subbundle of $\mathcal{E}_{\partial M}$ consisting of all $\eta \in \mathcal{E}_{\partial M}$ such that $\eta_{\alpha \beta}=0$ for $\alpha, \beta=1, \ldots, n-1$ and, in addition, $\eta_{n n}=0$. One could view every $\eta \in \mathcal{E}_{\partial M}$ as a bilinear form on $T_{\pi_{\mathcal{E}}(\eta)} M$. With this interpretation adopted, the subbundle $\mathcal{F}$ consists of those $\eta \in \mathcal{E}_{\partial M}$ that satisfy

$$
\eta(X, Y)=\eta(\hat{v}, \hat{v})=0
$$

for all $X$ and $Y$ tangent to $\partial M$ at $\pi_{\mathcal{E}}(\eta)$. Let $\mathcal{F}^{\perp}$ be the orthogonal complement of $\mathcal{F}$ in $\mathcal{E}_{\partial M}$.

\subsection{Proofs of the existence results}

We are now ready to prove Theorem 3.1. In order to do so, we will supplement the Ricci-DeTurck flow (3.3) with boundary conditions and an initial condition. Theorem 2.1 will then imply the existence of a solution. By modifying this solution, it is possible to obtain a Riemannian metric on $M$ that satisfies (3.1)-(3.2) and exhibits the desired boundary behavior.

Proof of Theorem 3.1. Recall that we are given a smooth real-valued function $\mu$ on $[0, \infty)$ with $\mu(0)=1$. Let us impose boundary conditions on the solutions of (3.3) by demanding that

$$
\begin{aligned}
\operatorname{Pr}_{\mathcal{F}} \bar{g}(x, t) & =o(x), \\
\overline{\mathrm{I}}_{\alpha \beta}(x, t) & =\frac{1}{2} \mu(t)\left(\bar{g}_{\alpha \gamma}(x, t) \hat{g}^{\gamma \sigma}(x) \hat{\mathrm{I}}_{\sigma \beta}(x)+\bar{g}_{\beta \gamma}(x, t) \hat{g}^{\gamma \sigma}(x) \hat{\mathrm{I}}_{\sigma \alpha}(x)\right), \\
P_{n}^{\bar{g}}(x, t) & =0, \quad x \in \partial M, t \in(0, T) .
\end{aligned}
$$


Here, $o$ is the zero section in $\mathcal{E}$, and $\overline{\mathrm{II}}$ is the second fundamental form field of $\partial M$ with respect to $\bar{g}$. A computation demonstrates that the boundary conditions (3.5) are equivalent to the formulas

$$
\begin{aligned}
\operatorname{Pr}_{\mathcal{F}} \bar{g}(x, t) & =o(x), \\
\operatorname{Pr}_{\mathcal{F}^{\perp}}\left(\bar{g}^{n n}(x, t)\left(\hat{g}_{n n}(x)\right)^{\frac{1}{2}} \hat{\nabla}_{n} \bar{g}(x, t)\right) & =\zeta(\bar{g}(x, t)), \quad x \in \partial M, t \in(0, T),
\end{aligned}
$$

where $\zeta$ is a map from the set $\left\{\eta \in \mathcal{E}_{\partial M} \mid \eta\right.$ is positive-definite $\}$ to the bundle $\mathcal{F}^{\perp}$. The components of the tensor $\zeta(\bar{g}(x, t))$ appear as

$$
\begin{aligned}
\zeta_{\alpha \beta}(\bar{g}(x, t))= & -\mu(t)\left(\hat{g}_{n n}(x) \bar{g}^{n n}(x, t)\right)^{\frac{1}{2}}\left(\bar{g}_{\alpha \gamma}(x, t) \hat{g}^{\gamma \sigma}(x) \hat{\mathrm{I}}_{\sigma \beta}(x)+\bar{g}_{\beta \gamma}(x, t) \hat{g}^{\gamma \sigma}(x) \hat{\mathrm{I}}_{\sigma \alpha}(x)\right) \\
& +\hat{g}_{n n}(x) \bar{g}^{n n}(x, t)\left(\bar{g}_{\alpha \gamma}(x, t) \hat{g}^{\gamma \sigma}(x) \hat{\mathrm{I}}_{\sigma \beta}(x)+\bar{g}_{\beta \gamma}(x, t) \hat{g}^{\gamma \sigma}(x) \hat{\mathrm{I}}_{\sigma \alpha}(x)\right), \\
\zeta_{n n}(\bar{g}(x, t))= & -2 \bar{g}_{n n}(x, t)\left(\mu(t)\left(\hat{g}_{n n}(x) \bar{g}^{n n}(x, t)\right)^{\frac{1}{2}} \hat{g}^{\alpha \beta}(x) \hat{\mathrm{I}}_{\alpha \beta}(x)-\bar{g}^{\alpha \beta}(x, t) \hat{\mathrm{I}}_{\alpha \beta}(x)\right),
\end{aligned}
$$

and $\zeta_{\alpha n}(\bar{g}(x, t))=0$ whenever $x \in \partial M$ and $t \in[0, T)$. As usual, the Greek indices vary from 1 to $n-1$.

We supplement (3.3) with the initial condition

$$
\bar{g}(x, 0)=\hat{g}(x), \quad x \in M .
$$

Theorem 2.1 and Remark 2.3 imply the existence of a number $T>0$ and a mapping $\bar{g}: M \times[0, T) \rightarrow \mathcal{E}$ such that the following statements hold:

1. The tensor $\bar{g}(x, t)$ is positive-definite for every $x \in M$ and $t \in[0, T)$. In other words, $\bar{g}$ is a Riemannian metric on $M$ depending on $t \in[0, T)$.

2. The mappings $\bar{g}$ and $\hat{\nabla} \bar{g}$ are continuous on $M \times[0, T)$. Furthermore, $\bar{g}$ is smooth on $M \times(0, T)$.

3. Equalities (3.4), (3.6), and (3.7) hold true.

Evidently, $\bar{g}$ must also solve (3.3) and satisfy the boundary conditions (3.5) along with the initial condition (3.7). We will write $\overline{\mathcal{H}}$ for the mean curvature of $\partial M$ with respect to $\bar{g}$. Our next step is to modify $\bar{g}$ by means of the DeTurck diffeomorphisms. Then $\bar{g}$ will become a decent solution of problem (3.1)-(3.2). Once we have that, the proof of the theorem will be easy to complete.

Consider a mapping $P_{\bar{g}}: M \times[0, T) \rightarrow T M$ defined by the formula

$$
P_{\bar{g}}^{i}(x, t)=\bar{g}^{i j}(x, t) P_{j}^{\bar{g}}(x, t), \quad x \in M, t \in[0, T) .
$$

It is clear from (3.5) that $P_{\bar{g}}^{n}(x, t)$ must equal 0 when $x \in \partial M$ and $t \in[0, T)$. In other words, $P_{\bar{g}}(x, t)$ is tangent to $\partial M$ as long as $x \in \partial M$. Let us look at the equation

$$
\frac{\partial}{\partial t} \psi(x, t)=-P_{\bar{g}}(\psi(x, t), t), \quad x \in M, t \in(0, T),
$$

for $\psi: M \times[0, T) \rightarrow M$. We supplement this equation with the initial condition

$$
\psi(x, 0)=x, \quad x \in M .
$$

The mapping $P_{\bar{g}}$ is continuous on $M \times[0, T)$ and smooth on $M \times(0, T)$. Also, $P_{\bar{g}}(\cdot, 0)$ is identically zero on $M$. Using these properties along with the fact that $P_{\bar{g}}(x, t)$ is tangent to $\partial M$ whenever $x \in \partial M$, we can prove the existence of a unique $\psi: M \times[0, T) \rightarrow M$ such that

1. The map $\psi$ is continuous on $M \times[0, T)$ and smooth on $M \times(0, T)$.

2. Equalities (3.8) and (3.9) hold true.

3. The map $\psi(\cdot, t)$ is a diffeomorphism from the manifold $M$ to itself for every $t \in[0, T)$. 
The reader may find relevant material in [8, Chapter 3, Section 3.1]. It is customary to call $\psi(\cdot, t)$ the DeTurck diffeomorphisms.

Given $t \in[0, T)$, define the Riemannian metric $g(\cdot, t)$ on $M$ as the pullback of $\bar{g}(\cdot, t)$ by $\psi(\cdot, t)$. One can then verify that the mapping $g: M \times[0, T) \rightarrow T^{*} M \otimes T^{*} M$ is a decent solution of problem (3.1)-(3.2) on the set $M \times[0, T)$; see, e.g., [8, page 81]. Moreover, for each $t \in[0, T)$, the second fundamental form field II $(\cdot, t)$ is equal to the pullback of $\overline{\mathrm{I}}(\cdot, t)$ by the restriction of $\psi(\cdot, t)$ to $\partial M$. Keeping this fact in mind, taking (3.5) into account, and remembering the hypotheses of the theorem, we compute the mean curvature $\mathcal{H}(x, t)$ and see that

$$
\mathcal{H}(x, t)=\overline{\mathcal{H}}(\psi(x, t), t)=\mu(t) \hat{\mathcal{H}}(\psi(x, t))=\mu(t) \mathcal{H}_{0}, \quad x \in \partial M, t \in[0, T) .
$$

The desired conclusion follows immediately.

It is time to prove Proposition 3.3. Again, we have to supplement the Ricci-DeTurck flow (3.3) with boundary conditions. The next step will be to apply Theorem 2.1 and obtain a solution. We will then modify this solution by means of the DeTurck diffeomorphisms.

Proof of Proposition 3.3. Let us add boundary conditions to (3.3) by demanding that

$$
\begin{aligned}
\operatorname{Pr}_{\mathcal{F}} \bar{g}(x, t) & =o(x), \\
\overline{\mathrm{I}}_{\alpha \beta}(x, t) & =\hat{\mathrm{I}}_{\alpha \beta}(x), P_{n}^{\bar{g}}(x, t)=0, \quad x \in \partial M, t \in(0, T) .
\end{aligned}
$$

As before, $\overline{\mathrm{II}}$ is the second fundamental form field of $\partial M$ with respect to $\bar{g}$. A computation shows that formulas (3.10) are equivalent to

$$
\begin{aligned}
\operatorname{Pr}_{\mathcal{F}} \bar{g}(x, t) & =o(x), \\
\operatorname{Pr}_{\mathcal{F}^{\perp}}\left(\bar{g}^{n n}(x, t)\left(\hat{g}_{n n}(x)\right)^{\frac{1}{2}} \hat{\nabla}_{n} \bar{g}(x, t)\right) & =\chi(\bar{g}(x, t)), \quad x \in \partial M, t \in(0, T),
\end{aligned}
$$

with $\chi$ acting from $\left\{\eta \in \mathcal{E}_{\partial M} \mid \eta\right.$ is positive-definite $\}$ to $\mathcal{F}^{\perp}$. The components of $\chi(\bar{g}(x, t))$ are

$$
\begin{aligned}
\chi_{\alpha \beta}(\bar{g}(x, t))= & -2\left(\hat{g}_{n n}(x) \bar{g}^{n n}(x, t)\right)^{\frac{1}{2}} \hat{\mathrm{I}}_{\alpha \beta}(x) \\
& +\hat{g}_{n n}(x) \bar{g}^{n n}(x, t)\left(\bar{g}_{\alpha \gamma}(x, t) \hat{g}^{\gamma \sigma}(x) \hat{\mathrm{I}}_{\sigma \beta}(x)+\bar{g}_{\beta \gamma}(x, t) \hat{g}^{\gamma \sigma}(x) \hat{\mathrm{I}}_{\sigma \alpha}(x)\right), \\
\chi_{n n}(\bar{g}(x, t))= & -2\left(\left(\hat{g}_{n n}(x) \bar{g}_{n n}(x, t)\right)^{\frac{1}{2}}-\bar{g}_{n n}(x, t)\right) \bar{g}^{\alpha \beta}(x, t) \hat{\mathrm{I}}_{\alpha \beta}(x),
\end{aligned}
$$

and $\chi_{\alpha n}(\bar{g}(x, t))=0$ when $x \in \partial M$ and $t \in[0, T)$. As in the proof of Theorem 3.1 we apply Theorem 2.1 and Remark 2.3 to obtain $T>0$ and $\bar{g}: M \times[0, T) \rightarrow \mathcal{E}$ such that

1. The tensor $\bar{g}(x, t)$ is positive-definite for every $x \in M$ and $t \in[0, T)$.

2. The mappings $\bar{g}$ and $\hat{\nabla} \bar{g}$ are continuous on $M \times[0, T)$. Also, $\bar{g}$ is smooth on $M \times(0, T)$.

3. Equalities (3.3) and (3.10) hold true, and $\bar{g}(x, 0)=\hat{g}(x)$ whenever $x \in M$.

It remains to bring the DeTurck diffeomorphisms into the picture and to modify $\bar{g}$ by means of these diffeomorphisms. The latter action will yield a decent solution of (3.1)-(3.2).

Following the same procedure as in the proof of Theorem 3.1, let us define the mapping $P_{\bar{g}}: M \times[0, T) \rightarrow$ $T M$ and construct the corresponding $\psi: M \times[0, T) \rightarrow M$. We introduce the Riemannian metric $g(\cdot, t)$ on $M$ as the pullback of $\bar{g}(\cdot, t)$ by $\psi(\cdot, t)$ for every $t \in[0, T)$. One verifies that $g: M \times[0, T) \rightarrow T^{*} M \otimes T^{*} M$ is a decent solution of (3.1)-(3.2) on $M \times[0, T)$. Moreover, $\mathrm{II}(\cdot, t)$ coincides with the pullback of II $(\cdot, t)$ by the restriction of $\psi(\cdot, t)$ to $\partial M$ for each $t \in[0, T)$. This fact, along with (3.10), implies the desired conclusions.

\section{Acknowledgements}

I would like to thank Xiaodong Cao, Leonard Gross, and Carlos Kenig for the productive discussions. I also express my gratitude to the anonymous referee for his/her insightful review of the paper and useful suggestions. 


\section{References}

[1] P. Acquistapace, B. Terreni, On quasilinear parabolic systems, Math. Ann. 282 (1988), 315-335.

[2] H. Amann, Quasilinear parabolic systems under nonlinear boundary conditions, Arch. Rational Mech. Anal. 92 (1986), 153-192.

[3] H. Amann, Dynamic theory of quasilinear parabolic equations - II. Reaction-diffusion systems, Diff. Int. Equ. 3 (1990), 13-75.

[4] M. Bailesteanu, X. Cao, A. Pulemotov, Gradient estimates for the heat equation under the Ricci flow, J. Funct. Anal. 258 (2010), 3517-3542.

[5] S. Brendle, Curvature flows on surfaces with boundary, Math. Ann. 324 (2002), 491-519.

[6] N. Charalambous, L. Gross, The Yang-Mills heat semigroup on three-manifolds with boundary, arXiv:1004.1639v1 [math.AP] (2010).

[7] X.-Z. Chen, T. Dong, Ricci deformation of a metric on a Riemannian manifold with boundary, J. Zhejiang Univ. Sci. Ed. 33 (2006), 496-499.

[8] B. Chow, D. Knopf, The Ricci flow: An introduction, Amer. Math. Soc., Providence, RI, 2004.

[9] B. Chow, P. Lu, L. Ni, Hamilton's Ricci flow, Amer. Math. Soc., Providence, RI, 2006.

[10] J.C. Cortissoz, The Ricci flow on the two ball with a rotationally symmetric metric, Russian Math. (Iz. VUZ) 51 (2007), no. 12, 30-51.

[11] J.C. Cortissoz, Three-manifolds of positive curvature and convex weakly umbilic boundary, Geom. Dedicata 138 (2009), 83-98.

[12] M. Giaquinta, G. Modica, Local existence for quasilinear parabolic systems under nonlinear boundary conditions, Ann. Mat. Pura Appl. 149 (1987), 41-59.

[13] M. Headrick, T. Wiseman, Ricci flow and black holes, Class. Quantum Grav. 23 (2006), 6683-6707.

[14] G. Holzegel, T. Schmelzer, C. Warnick, Ricci flows connecting Taub-Bolt and Taub-NUT metrics, Class. Quantum Grav. 24 (2007), 6201-6217.

[15] O.A. Ladyženskaja, V.A. Solonnikov, N.N. Ural'ceva, Linear and quasilinear equations of parabolic type, Amer. Math. Soc., Providence, RI, 1968.

[16] T. Li, The Ricci flow on surfaces with boundary, Ph.D. Dissertation, University of California at San Diego, 1993.

[17] J. Morgan, G. Tian, Ricci Flow and the Poincaré Conjecture, Amer. Math. Soc., Providence, RI; Clay Mathematics Institute, Cambridge, MA, 2007.

[18] T.A. Oliynyk, V. Suneeta, E. Woolgar, A gradient flow for worldsheet nonlinear sigma models, Nuclear Phys. B 739 (2006), 441-458.

[19] T.A. Oliynyk, E. Woolgar, Rotationally symmetric Ricci flow on asymptotically flat manifolds, Comm. Anal. Geom. 15 (2007), 535-568.

[20] A. Pulemotov, The Li-Yau-Hamilton estimate and the Yang-Mills heat equation on manifolds with boundary, J. Funct. Anal. 255 (2008), 2933-2965.

[21] M. Sánchez, Geodesic connectedness of semi-Riemannian manifolds, Nonlinear Anal. 47 (2001), 30853102.

[22] Y. Shen, New results on some dynamical and stationary problems in geometry, Ph.D. Dissertation, Stanford University, 1992. 
[23] Y. Shen, On Ricci deformation of a Riemannian metric on manifold with boundary, Pacific J. Math. 173 (1996), 203-221.

[24] W.-X. Shi, Ricci deformation of the metric on complete noncompact Riemannian manifolds, J. Differential Geom. 30 (1989), 303-394.

[25] M. Simon, Deformation of $C^{0}$ Riemannian metrics in the direction of their Ricci curvature, Comm. Anal. Geom. 10 (2002), 1033-1074.

[26] M. Simon, Deforming Lipschitz metrics into smooth metrics while keeping their curvature operator non-negative, Geometric evolution equations, Amer. Math. Soc., Providence, RI, 2005, 167-179.

[27] V.A. Solonnikov, On boundary value problems for linear parabolic systems of differential equations of general form, Proc. Steklov Inst. Math. 83 (1965), 1-184.

[28] B. Terreni, Nonhomogeneous initial-boundary value problems for linear parabolic systems, Studia Math. 92 (1989), 141-175.

[29] P. Topping, Lectures on the Ricci flow, Cambridge University Press, Cambridge, 2006.

[30] P. Weidemaier, Local existence for parabolic problems with fully nonlinear boundary condition; an $L_{p^{-}}$approach, Ann. Mat. Pura Appl. 160 (1991), 207-222. 